\title{
REPRESENTATIONS OF SPACES AS FUNCTION SPACES
}

\author{
by M. P. STANNETT $\dagger$
}

(Received 12 September, 1986)

0. Introduction. Given a topological space $X$, we can consider the group $G(X)$ of all autohomeomorphisms of $X$. Much is known about the relationship between $X$ and $G(X)$ for certain restricted classes of the space $X$; Whittaker [7] has shown that the existence of an isomorphism between any two sufficiently large subgroups of $G(X)$ and $G(Y)$ implies that $X$ and $Y$ are actually homeomorphic, whenever these are both compact, locally Euclidean manifolds, with or without boundary; Fine and Schweigert [1] give a detailed analysis of $G(\mathbb{R})$; recently, Neumann [4], Mekler [3] and Truss [6] have considered in depth the group $G(\mathbb{Q})$.

A proven technique when studying arbitrary spaces is to embed them within other spaces about which more is known; thus the study of compact Hausdorff spaces allows for a greater understanding of Tychonov spaces (i.e. those spaces which occur as subspaces of compact Hausdorff spaces). Similarly, Shimrat [5] has shown that every space $X$ can be embedded in a homogeneous superspace.

We shall show that every space $X$ embeds as a retract within the space $C(G(X), X)$ of continuous functions from $G(X)$ into $X$ (with suitably defined topologies), and that this embedding has the additional property that every autohomeomorphism of $X$ extends to an autohomeomorphism of $C(G(X), X)$. Moreover, if $X$ is Tychonov, so is $C(G(X), X)$, and our retraction extends to a retraction of $\beta C(G(X), X)$ onto $\beta X$.

1. A class of topologies for $G(X)$. Let $X$ be some fixed but arbitrary topological space. Throughout this paper, we denote by $p$ any topological property satisfying

(i) $p(\{x\}) \forall x \in X$,

(ii) $[p(A)$ and $p(B)] \Rightarrow p(A \cup B) \forall A, B \in X$.

For example, $p(A)$ might mean " $A$ is compact", " $A$ is finite", or even " $A \subseteq X$ ". We shall write $(p ; B)$ to mean that $p(A)$ holds for every relatively closed subset $A \subseteq B \subseteq X$.

If $A \subseteq X$, we denote by $G_{A}$ the stabiliser of $A$ in $G(X)$, viz:

$$
G_{A}=\{g \in G(X): g(a)=a \forall a \in A\} .
$$

Given some such property $p$, we may take $\mathbb{F}_{p}=\left\{G_{A}: A \subseteq X, p(A)\right\}$ to be a fundamental system of open sets for $G(X)$. For we need only check that

(i) if $U, V \in \mathbb{F}_{p}$, then there exists $W \in \mathbb{F}_{p}$ such that $W \subseteq U \cap V$;

(ii) if $U \in \mathbb{F}_{p}, g \in G(X)$, then there exists $V \in \mathbb{F}_{p}$ such that $g^{-1} V g \subseteq U$. See e.g. $[1$, p. 28].

Now (i) follows by taking $W=G_{A \cup B}$, where $U=G_{A}$ and $V=G_{B}$, recalling our initial

† The author would like to acknowledge the support of the SERC during the preparation of this paper.

Glasgow Math. J. 30 (1988) 189-193. 
hypothesis that $p(A)$ and $p(B)$ together imply $p(A \cup B)$. Condition (ii) is satisfied since every conjugate of a stabiliser is again a stabiliser, and because $p$ is preserved under maps in $G(X)$, since it is topological. This gives a topology on $G(X)$ with which it becomes a topological group, which we shall denote $G_{p}(X)$. When $p$ is the particular property of being compact, we write $G_{K}(X)$ instead of $G_{p}(X)$.

LeMMA 1. $G_{p}(X)$ is a zero-dimensional Tychonov space.

Proof. If $g \in \cap \mathbb{F}_{p}$, then $g \in G_{A}$ for every $A \subseteq X$ satisfying $p(A)$. In particular, $g \in G_{\{x\}}$ for every $x \in X$, whence $g=1$. So $G_{p}(X)$ is $T_{1}$ and so Hausdorff. It is now sufficient to note that every element of $\mathbb{F}_{p}$ is clopen, since its complement is a union of its cosets, each of which is open.

When discussing a topological group $G$, it is often of interest to determine whether $G$ is locally compact, since we may then define Haar measure on $G$. Note that, by taking $p(A) \equiv$ " $A \subseteq X$ ", we can always ensure that $G_{p}(X)$ is discrete; but for other properties $p$, $G_{p}(X)$ need not be locally compact, as we now demonstrate.

If $G_{p}(X)$ is locally compact, for some property $p$, then Lemma 1 tells us that the group identity, 1 , has a basis of compact clopen sets, each in $\mathbb{F}_{p}$.

We are grateful to the referee for greatly improving upon our original proof of the next result.

Theorem 2. Let $A \subseteq B \subseteq X$, where $p(A)$ and $(p ; B)$. Suppose $G_{A}$ is compact in $G_{p}(X)$. Then there exists a finite $S \subseteq B \backslash A$ such that

$$
G_{B}=G_{\overline{A \cup S}}{ }^{B}
$$

Proof. For each finite subset $F$ of $B \backslash A$ define $A_{F}=\overline{A \cup F}^{B}$ and $H_{F}=G_{A_{F}} \backslash G_{B}$. Then $H_{F}$ is a closed subset of the compact set $G_{A} \backslash G_{B}$. Since the union of the $A_{F}$ 's is $B$, we have $\cap H_{F}=\varnothing$, and so the $H_{F}$ 's fail to have the finite intersection property. Hence we can find finite sets $F_{1}, \ldots, F_{n}$ in $B \backslash A$ and

$$
\varnothing=H_{F_{1}} \cap \ldots \cap H_{F_{n}}=H_{F_{1} \cup \ldots \cup F_{n}}
$$

So, putting $S=F_{1} \cup \ldots \cup F_{n}$ completes the proof.

Corollary 3. $G_{K}(\mathbb{Q})$ is not locally compact.

Proof. Suppose that $G_{A}$ were compact, for some compact $A \subseteq \mathbb{Q}$. Let $T$ be any infinite compact subset of $\mathbb{Q}$, disjoint from $A$. Taking $B=A \cup T$ satisfies the conditions of the theorem, whence there exists some finite $S \subseteq T$ such that $G_{A \cup S}=G_{A \cup T}$ (since $\overline{A \cup S}^{A \cup T}=A \cup S$ ). But this is clearly nonsense. For, choose any $x \in T \backslash S$, and any interval $(a, b)$ containing $x$ and disjoint from $A \cup S$. Then we can easily find an element of $G(\mathbb{Q})$ fixing $\mathbb{Q} \backslash(a, b)$ and moving $x$.

2. Representing spaces as function spaces. We conclude this paper by showing that every space $X$ may be regarded as a space of continuous functions from $G_{p}(X)$ into $X$. Our method parallels that of the embedding of a vector space in its second dual. 
Lemma 4. Given $x \in X$, define $\phi_{x}: G_{p}(X) \rightarrow X$ by $\phi_{x}(g)=g(x)$. Then $\phi_{x}^{-1}(S)$ is open in $G_{p}(X)$ for every $S \subseteq X$.

Proof. We simply note that $\phi_{x}^{-1}(S)=\phi_{x}^{-1}(S) . G_{\{x\}}$, and the latter is open since $G_{\{x\}} \in \mathbb{F}_{p}$.

Consequently, we may sensibly define a map $\Phi: X \rightarrow C\left(G_{p}(X), X\right)$ by $\Phi(x)=\phi_{x}$ for all $x \in X$. The map $\Phi$ is injective, since $\phi_{x}=\phi_{y}$ implies $x=\phi_{x}(1)=\phi_{y}(1)=y$.

Let $C\left(G_{p}(X), X\right)$ be given the finite-open topology. That is, we take as a subbase for the topology all sets of the form

$$
(K, U)=\left\{f \in C\left(G_{p}(X), X\right): f(K) \subseteq U\right\},
$$

where $K$ ranges over all finite subsets of $G(X)$, and $U$ over all open subsets of $X$. We shall always assume that $C\left(G_{p}(X), X\right)$ is equipped with this topology.

THEOREM 5. The map $\Phi: X \rightarrow C\left(G_{p}(X), X\right)$ is a topological embedding.

Proof. We have already seen that $\Phi$ is injective, so we need only demonstrate bicontinuity. If $(K, U)$ is a typical subbasic set in $C\left(G_{p}(X), X\right)$, then

$$
\begin{aligned}
\Phi^{-1}(K, U) & =\left\{x \in X: \phi_{x}(K) \subseteq U\right\} \\
& =\{x \in X: g(x) \in U \forall g \in K\} \\
& =\bigcap\left\{g^{-1}(U): g \in K\right\} .
\end{aligned}
$$

The latter is a finite intersection of open sets, and so is open in $X$.

Conversely, if $U$ is an open set in $X$, then

$$
\begin{aligned}
\Phi(U) & =\left\{\phi_{x}: x \in U\right\} \\
& =\left\{f \in C\left(G_{p}(X), X\right): f(1) \in U\right\} \cap \operatorname{im} \Phi \\
& =(\{1\}, U) \cap \operatorname{im} \Phi,
\end{aligned}
$$

which is open in im $\Phi$, since $(\{1\}, U)$ is open in $C\left(G_{p}(X), X\right)$.

THEOREM 6. Let $g \in G(X)$. Then $g$ has an extension $\psi_{g} \in G\left(C\left(G_{p}(X)\right.\right.$, $\left.\left.X\right)\right)$, i.e. $g=\left.\psi_{g}\right|_{X}$, where we identify $X$ and $\Phi(X)$.

Proof. Define $\psi_{g}: C\left(G_{p}(X), X\right) \rightarrow C\left(G_{p}(X), X\right)$ by $\psi_{g}(f)(\tilde{g})=f(\tilde{g} g)$ for all $g \epsilon$ $G(X), f \in C\left(G_{p}(X), X\right)$. We shall show that $\psi_{g} \in G\left(C\left(G_{p}(X), X\right)\right)$.

Note first that $\psi_{g}(f) \in C\left(G_{p}(X), X\right)$ whenever $g \in G(X)$ and $f \in C\left(G_{p}(X), X\right)$. For suppose $U \subseteq X$ is open. Then

$$
\begin{aligned}
{\left[\psi_{g}(f)\right]^{-1}(U) } & =\left\{\tilde{g}: \psi_{g}(f)(\tilde{g}) \in U\right\} \\
& =\{\tilde{g}: f(\tilde{g} g) \in U\} \\
& =\left\{\tilde{g}: \tilde{g} g \in f^{-1}(U)\right\} \\
& =f^{-1}(U) \cdot g^{-1},
\end{aligned}
$$

which is open, since $f^{-1}(U)$ is open in $G_{p}(X)$ by the continuity of $f$. 
Now suppose that $\psi_{g}\left(f_{1}\right)=\psi_{g}\left(f_{2}\right)$. Then given any $\tilde{g} \in G(X)$, we have $f_{1}(\tilde{g} g)=f_{2}(\tilde{g} g)$. Since $\tilde{g} g$ ranges over all of $G(X)$ as $\tilde{g}$ does, we see that $f_{1}=f_{2}$. So $\psi_{g}$ is injective.

Moreover, if $(K, U)$ is a typical subbasic open set in $C\left(G_{p}(X), X\right)$, then

$$
\begin{aligned}
\psi_{g}^{-1}(K, U) & =\left\{f: \psi_{g}(f)(K) \subseteq U\right\} \\
& =\{f: f(\tilde{g} g) \in U \forall \tilde{g} \in K\} \\
& =(K g, U)
\end{aligned}
$$

which is open. So $\psi_{g}$ is continuous.

Likewise, $\psi_{g^{-1}}$ is an injective continuous map, and since $\psi_{g} \circ \psi_{g^{-1}}=\psi_{g^{-1}} \circ \psi_{g}=$ id, we see that $\psi_{g}$ is a homeomorphism, as claimed.

It remains only to show that $\psi_{g}$ extends $g$. But this is clear, since $\psi_{g}\left(\phi_{x}\right)(\tilde{g})=$ $\phi_{x}(\bar{g} g)=\tilde{g} g(x)=\phi_{g(x)}(\tilde{g})$. Thus $\Phi \circ g=\psi_{g} \circ \Phi$, as required.

We now show that $X$ is a retract of $C\left(G_{p}(X), X\right)$, and that, if $X$ is Tychonov, then so is $C\left(G_{p}(X), X\right)$. We obtain, as a corollary, that $\beta X=\bar{X}^{\beta C\left(G_{p}(X), X\right)}$ whenever $X$ is Tychonov.

LEMMA 7. $X$ is a retract of $C\left(G_{p}(X), X\right)$.

Proof. Define $\theta: C\left(G_{p}(X), X\right) \rightarrow X$ by $\theta(f)=f(1)$. If $x=X$, then $\theta\left(\phi_{x}\right)=\phi_{x}(1)=x$, so that $X$ is fixed by $\theta$. To show that $\theta$ is continuous, we consider a typical open set $U$ of $X$. Now

$$
\begin{aligned}
\theta^{-1}(U) & =\left\{f \in C\left(G_{p}(X), X\right): \theta(f) \in U\right\} \\
& =(\{1\}, U),
\end{aligned}
$$

which is open in $C\left(G_{p}(X), X\right)$.

Lemma 8. If $X$ is Tychonov, so is $C\left(G_{p}(X), X\right)$.

Proof. Let $f \in(K, U)$, where $(K, U)$ is some typical subbasic open set in $C\left(G_{p}(X), X\right)$. We have to find a continuous $F: C\left(G_{p}(X), X\right) \rightarrow[0,1]$, such that $F(f)=0$ and $F \equiv 1$ outside $(K, U)$ (see e.g. $[8,14.8])$.

The set $f(K)$ is a finite subset of $U$. Since $X$ is Tychonov, there exists a continuous $\hat{F}: X \rightarrow[0,1]$ such that $\hat{F} \equiv 0$ on $f(K)$ and $\hat{F} \equiv 1$ outside $U$. Define $F: C\left(G_{p}(X), X\right) \rightarrow$ $[0,1]$ by

$$
F(\tilde{f})=\max \{\hat{F}(\tilde{f}(g)): g \in K\} .
$$

Then $F(f)=0$, while if $\tilde{f} \in(K, U)$, then $\tilde{f}(g) \notin U$ for some $g \in K$, whence $F(\tilde{f})=1$.

We now show that $F$ is continuous. Write $K=\left\{g_{1}, \ldots, g_{n}\right\}$ and for each $i=$ $1, \ldots, n$, define $\psi_{i}: C\left(G_{p}(X), X\right) \rightarrow X$ by $\psi_{i}(\tilde{f})=\tilde{f}\left(g_{i}\right)$. Then $\psi_{i}=\theta \circ \psi_{g_{i}}$, so $\psi_{i}$ is continuous for each $i=1, \ldots, n$, where $\theta$ is as in Lemma 7 and $\phi_{g_{i}}$ as in Theorem 6. It is now enough to note that

$$
\begin{aligned}
F(\tilde{f}) & =\max \{\hat{F}(\tilde{f}(g)): g \in K\} \\
& =\max \left\{\hat{F} \circ \psi_{i}(\tilde{f}): i=1, \ldots, n\right\} .
\end{aligned}
$$

Since each $F \circ \psi_{i}$ is continuous, so is their maximum. 
We conclude the proof by showing that $C\left(G_{p}(X), X\right)$ is Hausdorff. If $f_{1}, f_{2}$ are distinct elements of $C\left(G_{p}(X), X\right)$, there exists some $g \in G(X)$ such that $f_{1}(g) \neq f_{2}(g)$. Since $X$ is Hausdorff, there exist disjoint open sets $U, V$ with $f_{1}(g) \in U$ and $f_{2}(g) \in V$. Now $f_{1} \in(\{g\}, U), f_{2} \in(\{g\}, V)$, and these two open sets are disjoint.

Corollary 9. Let $X$ be Tychonov. Then

$$
\beta X=\bar{X}^{\beta C\left(G_{p}(X), X\right)}
$$

and, moreover, $\theta$ extends to a retraction $\theta^{\beta}: \beta\left(C\left(G_{p}(X), X\right) \rightarrow \beta X\right.$.

Proof. According to Lemma $8, C\left(G_{p}(X), X\right)$ is Tychonov, so that $\beta C\left(G_{p}(X), X\right)$ exists. Now $X$ is a retract of, and so is $C^{*}$-embedded in, $C\left(G_{p}(X), X\right)$. Hence $X$ is $C^{*}$-embedded in its compactification, $\bar{X}^{\beta C\left(G_{p}(X), X\right)}$, whence $\beta X=\bar{X}^{\beta C\left(G_{p}(X), X\right)}$.

Now $\theta$ has a natural extension $\theta^{\beta}: \beta C\left(G_{p}(X), X\right) \rightarrow \beta X$; since $\theta$ acts as the identity on $X, \theta^{\beta}$ must act as the identity on $\bar{X}^{\beta C\left(G_{p}(X), X\right)}=\beta X$, as claimed.

\section{REFERENCES}

1. N. J. Fine and G. E. Schweigert, On the group of homeomorphisms of an arc, Ann. of Math. (2) 62 (1955), 237-253.

2. P. J. Higgins, An introduction to topological groups, London Mathematical Society Lecture Note Series 15 (Cambridge, 1979).

3. A. H. Mekler, Groups embeddable in the autohomeomorphisms of $\mathbb{Q}, J$. London Math. Soc. (2) 33 (1986), 49-58.

4. P. M. Neumann, Automorphisms of the rational world, J. London Math. Soc. (2) 32 (1985), 439-448. 304-311.

5. M. Shimrat, Embedding in homogeneous spaces, Quart, J. Math. Oxford Ser. (2) 5 (1954),

6. J. K. Truss, Embeddings of infinite permutation groups, Proceedings of Groups 1985 at St Andrews (to appear).

7. J. V. Whittaker, On isomorphic groups and homeomorphic spaces, Ann. of Math. (2) 78 (1963), 74-91.

8. S. Willard, General Topology, (Addison-Wesley, 1970).

Department of Computer Science

THE UNIVERSITY

SHEFFIELD

S3 7RH 